Cahiers « Mondes anciens »

MONDES

ANCIENS

Histoire et anthropologie des mondes anciens

$6 \mid 2015$

Mères grecques

\title{
Transmission maternelle en Grèce ancienne : du physique au comportement
}

Maternal Transmission in Ancient Greece: From the Body to the Behaviour

\section{Florence Gherchanoc}

\section{(2) OpenEdition}

Journals

Édition électronique

URL : http://journals.openedition.org/mondesanciens/1315

DOI : 10.4000/mondesanciens. 1315

ISSN : 2107-0199

Éditeur

UMR 8210 Anthropologie et Histoire des Mondes Antiques

Référence électronique

Florence Gherchanoc, «Transmission maternelle en Grèce ancienne : du physique au

comportement », Cahiers « Mondes anciens » [En ligne], 6 | 2015, mis en ligne le 27 janvier 2015,

consulté le 20 avril 2019. URL : http://journals.openedition.org/mondesanciens/1315; DOI : 10.4000/ mondesanciens. 1315

Ce document a été généré automatiquement le 20 avril 2019.

\section{(1) ()}

Les Cahiers «Mondes Anciens » sont mis à disposition selon les termes de la licence Creative Commons Attribution - Pas d'Utilisation Commerciale - Pas de Modification 4.0 International. 


\title{
Transmission maternelle en Grèce ancienne : du physique au comportement
}

\author{
Maternal Transmission in Ancient Greece: From the Body to the Behaviour
}

Florence Gherchanoc

1 Dans le Complexe de Zeus. Représentations de la paternité en Grèce ancienne, paru en 2004, Jean-Baptiste Bonnard a mis en perspective les fonctions d'une survalorisation de la paternité, dans des sociétés où les mères, filles et épouses de citoyens, sont déjà tenues à l'écart du pouvoir politique et des rôles sociaux les plus importants. Son analyse des rapports de force entre une paternité quasi exclusive et une maternité passive et dépréciée l'a conduit à insister sur le rôle "fondateur " du père dans la reproduction humaine, à la fois dans la fabrication et l'éducation des enfants.

2 Sans nier et minimiser la place de la figure paternelle, en particulier à l'époque classique, je voudrais, dans cet article, réévaluer néanmoins celle de la mère en proposant quelques pistes de réflexion sur les caractères physiques et les comportements que celle-ci transmet à ses enfants, filles et garçons. Car, pour certains auteurs, la mère n'est pas seulement conçue comme un réceptacle de la semence paternelle - même si c'est l'opinion d'Aristote - ni non plus comme une simple médiatrice du père à l'enfant : d'une noble ascendance, d'un statut ou d'un patrimoine.

3 Aussi, en confrontant des sources variées - textes médicaux et biologiques, tragédies et comédies, plaidoyers, poésie -, mon article proposera-t-il de réfléchir à la part de la mère dans la construction de l'identité de l'enfant. En d'autres termes, suivant des logiques génériques et narratives singulières, dans les représentations collectives des anciens Grecs, en particulier à l'époque classique, quelle « empreinte » laisserait une mère sur ses enfants, les marquant durablement dans leur chair comme dans leur attitude? En quoi cela aurait-il une influence sur leurs rôles sociaux et/ou politiques dans la cité?

Même si la transmission est fondée sur la biologie, on verra donc comment les théories biologiques de la reproduction, précisément les discours sur cette transmission de la 
mère aux enfants - les caractères de l'hérédité -, relèvent d'une construction culturelle propre à une période historique donnée ${ }^{1}$.

\section{Caractéristiques et ressemblances physiques ${ }^{2}$}

\section{Détermination du sexe biologique}

On le sait, en Grèce ancienne, le rôle de la mère dans la détermination du sexe de l'embryon est sous-évalué jusqu'aux médecins hippocratiques, vers la fin du ve siècle av. n. è. Pour certains de ces derniers, en effet, contrairement à leurs prédécesseurs, les semences des deux parents transmettent des informations au fœetus durant la conception, justifiant des caractéristiques et des ressemblances physiques. Ils reconnaissent notamment l'existence d'une semence féminine active dans le processus de la génération et dans la détermination du sexe de l'embryon, en fonction de son epikrateia ou domination (liée à sa quantité) :

La semence vient dans la matrice du corps entier de la femme et de l'homme, faible des parties faibles, forte des parties fortes. Ces qualités sont fatalement données aussi à l'enfant.

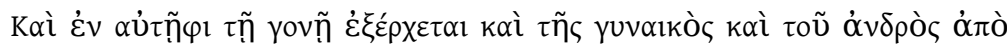

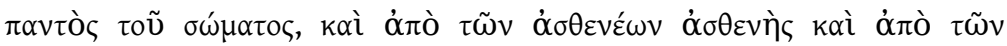

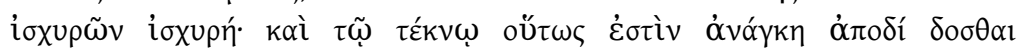
(Hippocrate, De la génération, VIII, 1).

6 En revanche, Aristote (à l'instar des Présocratiques) défend des conceptions différentes où la part faite au père dans la génération et dans la transmission des caractères de l'hérédité (sexe et aspect physique) est totale. Le ventre maternel est compris comme le lieu de gestation du fotus où s'opère cette détermination. La mère n'est qu'un réceptacle passif (Bonnard 2004, p. 144 et 190-191):

Voilà pourquoi, partout où cela est possible et dans la mesure où cela est possible, le mâle est distinct de la femelle. Car le principe de mouvement (archê tês kinêseôs), c'est-à-dire le mâle, est pour les êtres qui naissent ce qu'il y a de meilleur et de plus divin, tandis que la femelle est la matière (hulê) (Aristote, Génération des animaux, II, 1, 732a6-9).

7 La puissance de la semence paternelle agit pleinement sur la matière maternelle et des garçons naissent. Dans le cas contraire, celui où l'action de la puissance de la semence paternelle sur la matière est défaillante, en particulier chez les jeunes gens ou les vieillards, il naît des filles (Génération des animaux, IV, 3, 767b8)4. Dans tous les cas, l'enfant est le produit de son père.

\section{Traits et marques physiques}

8 La question de la ressemblance physique oppose également les médecins de Cos et le philosophe de Stagire.

D'après le corpus hippocratique, l'enfant ressemble aux deux parents :

Il n'est pas possible de ressembler en tout à la mère et en rien au père ou le contraire, ni non plus de ne ressembler en rien à aucun des deux; au contraire, il est nécessaire de ressembler à tous les deux en quelque chose, puisque la semence vient des deux à l'enfant. 


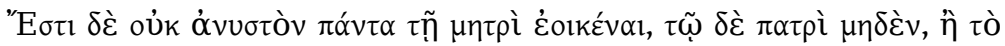

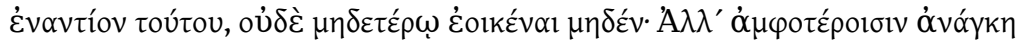

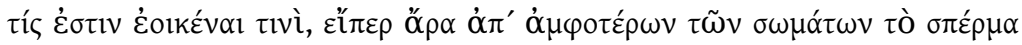

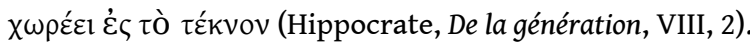

10 Chacun d'eux, la femme et l'homme, possède à la fois la semence mâle et femelle. Chaque parent émet suivant les cas l'une ou l'autre semence, ce qui détermine et répartit les ressemblances entre père et mère. Suivant leur mélange et selon que la semence mâle (forte) ou femelle (faible) l'emporte, l'enfant est garçon ou fille et a plus ou moins les traits de chacun de ses deux parents (cf. Hippocrate, De la Génération, VI, 1-2). Il est donc plus ou moins viril et fort ou plus ou moins féminin et beau :

Si donc les cellules émises par les deux partenaires se trouvent être mâles, elles croissent selon ce qui est à leur disposition et deviennent des hommes à l'âme

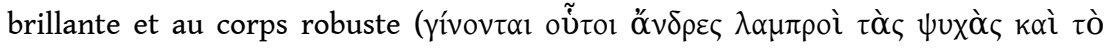
$\sigma \omega \tilde{\mu \alpha}$ i $\sigma \chi \chi \rho \rho i)$, si du moins ils ne sont pas gâtés par le régime subséquent. 3. Si la sécrétion de l'homme est mâle et celle de la femme femelle, mais que le mâle l'emporte, l'âme plus faible se mélange à la plus forte, car il ne se trouve alors rien de plus apparenté à elle vers quoi elle pourrait se retirer. Car la plus petite va vers la plus grande et la plus grande vers la plus petite. Et ensemble, elles maîtrisent ce qui se trouve à leur disposition; l'élément mâle se développe et l'élément femelle dépérit et se sépare pour aller vers un autre destin. Ces hommes sont moins brillants que les précédents ; cependant, puisque l'élément mâle venant de l'homme l'a emporté, ils sont virils ( $\alpha \dot{\alpha} \delta \rho \varepsilon \tilde{o}_{\mathrm{o}}$ ) et portent ce nom à juste titre. 4 . Si l'élément mâle vient de la femme et l'élément femelle de l'homme, mais que le mâle l'emporte, il se développe de la même façon que précédemment et l'élément femelle dépérit. Ces hommes sont efféminés (’̊ $v \delta \rho o ́ \gamma v v o l)$ et sont appelés ainsi à juste titre. Voilà les trois façons dont se forment les hommes; leur degré de virilité diffère, selon la teneur en eau et en feu des cellules, le régime, l'éducation, les habitudes [...] XXIX. 1. La femme se forme de la même façon. Si l'élément femelle est sécrété par

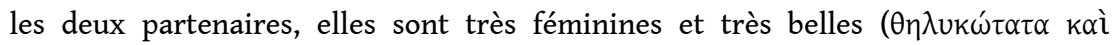

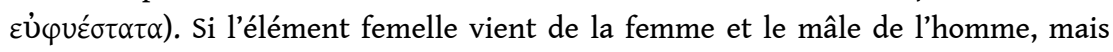

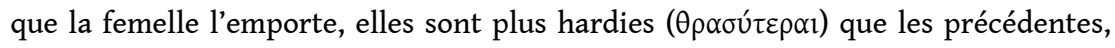
tout en restant modérées, elles aussi. Si l'élément femelle vient de l'homme et l'élément mâle de la femme, mais que le femelle l'emporte, il se développe de la

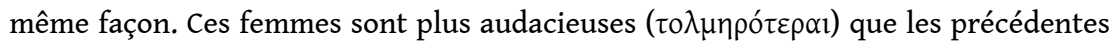
et sont appelées viriles ( $\dot{\alpha} v \delta \rho \varepsilon \tilde{\alpha} \alpha)$ (Hippocrate, Du régime, I, XXVIII, 2-XXIX, 1)5.

11 Ce qui est déterminant, c'est la répartition du masculin et du féminin entre semences paternelle et maternelle et non la part ou contribution de la mère. Aussi la plupart des auteurs anciens qui reconnaissent l'existence d'une semence féminine considèrent-ils que l'enfant prend le sexe et ressemble d'aspect au parent dont la semence a été dominante (Roux 2009, p. 311).

12 En revanche, pour Aristote, le meilleur modèle est celui où « le fils ressemble au père et la fille à la mère » car ne pas ressembler à ses parents, c'est aussi déjà une sorte de monstruosité, une déviance de la nature (cf. Aristote, Génération des animaux, IV, 3, 1-2. 768a22-23 ; cf. aussi Histoire des animaux, VII, 6, 586a4-6 ; Politique, II, III, 8-9 1262a14-24 ; Pline, VII, 51 ; voir Bonnard 2013, p. 26). Dans ce processus de génération, la «femme apporte sa contribution dans le seul sang menstruel qui est sa véritable semence et qui constituera la matière de l'enfant» (Roux 2009, p. 310). Aussi la mère exclue de la détermination du sexe de l'embryon l'est-elle encore largement de la transmission des caractères à l'enfant. Tout dépend de l'action de la semence paternelle et de son efficience comme de sa déficience (cf. infra). 


\section{Modelage et influences maternels} processus de conception et durant la première enfance, qui dépasse la question de l'existence ou non d'une semence féminine active. Par exemple, d'après Platon, «[1]a femme enceinte se promènera ( $\pi \varepsilon \rho \iota \pi \alpha \tau \varepsilon \tau v)$; elle modèlera son nouveau-né comme une

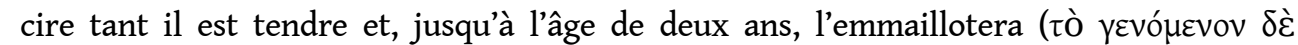

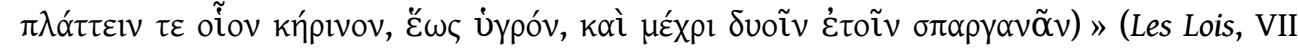
789e). La mère joue donc un rôle non négligeable dans la «fabrication" de l'enfant compris comme un modelage d'abord corporel. Être en maturation et perméable, le bébé reçoit d'elle des éléments qui forgent, structurent et marquent son enveloppe physique puis son esprit et son caractère.

Quelques textes permettent de mieux caractériser cette action maternelle sur le fotus, puis sur le nourrisson. Précisément, ils explicitent comment la mère, par son état d'esprit comme par son corps et ses humeurs, communique des troubles psychiques, des signes physiques, voire des maladies à sa progéniture.

Enceinte, la mère imprimerait sa marque sur le nouveau-né, en premier lieu, en raison des émotions qui l'agitent, de ses impressions sensorielles et de son imagination. Celles-ci agiraient pendant la grossesse et pourraient laisser des traces bonnes ou mauvaises sur le bébé en gestation. À cet égard, Platon préconise, quand il réfléchit aux institutions de sa cité crétoise, de veiller particulièrement aux femmes enceintes pour qu'elles ne connaissent aucun excès ni de bonheur ni de peine : «pour conserver l'humeur sereine,

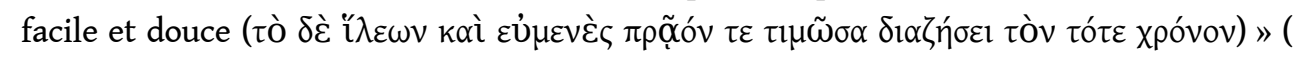
Les Lois, VII 792e). Cette idée relative aux dispositions d'esprit maternelles est également développée par Aristote. Même si, pour ce dernier, le père détient une archê qui est moteur dans la détermination du sexe de l'embryon et des caractères de l'enfant, la mère transmet cependant ses sentiments, sensations, idées et pensées au foetus comme une terre ${ }^{6}$ qui, suivant sa richesse et sa qualité, nourrit et contribue à la bonne ou mauvaise croissance des végétaux :

Il faut aussi que les femmes enceintes prennent soin de leur corps: elles ne resteront pas inactives et ne se contenteront pas d'une nourriture peu substantielle. [...] Leur esprit toutefois, au contraire de leur corps, aura avantage à vivre d'une manière moins active, car les enfants avant leur naissance subissent évidemment l'influence de celle qui les porte, comme les plantes, celle de la terre.

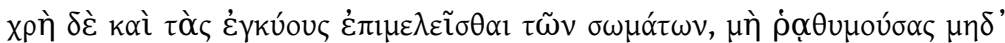

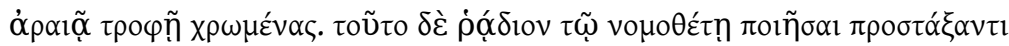

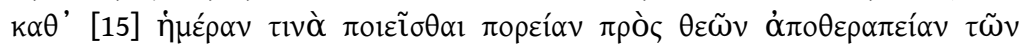

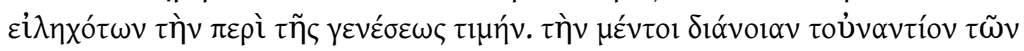

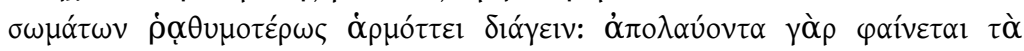

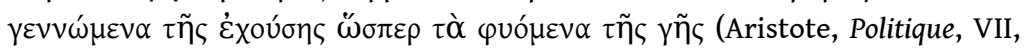
$14,1335 b)$.

Des théories médicales comparables sont développées au sujet de l'imprégnation par le regard. Ainsi, notamment, pour Soranos d'Éphèse, médecin du II $\mathrm{e}$. de $n$. è, héritier d'une tradition et d'un savoir antérieurs :

Il faut aussi dire que tel ou tel état d'âme (tês psuchês katastêma) apporte des changements dans les caractères (tupoi) du fotus. Ainsi des femmes qui, au cours d'un rapprochement sexuel, avaient vu des singes ont accouché d'êtres simiesques; 
le tyran de Chypre, qui était contrefait, forçait sa femme à contempler, pendant les rapports, des statues admirables : il fut le père de beaux enfants (eumorphôn paidôn )! [...] Pour éviter donc que le fotus ne se forme sous des apparences hideuses, en raison des images étranges qui s'imposent à l'esprit dans l'ivresse, les femmes doivent arriver sobres au rapport sexuel ; de plus, il existe entre les mères et leurs enfants une certaine ressemblance (homoiotês) non pas seulement physique (kata sôma) mais aussi morale (kata psuchên); aussi est-il bon que le fœtus soit façonné à la ressemblance d'un esprit équilibré et non à celle d'un esprit que l'ivresse fait divaguer... (Soranos d'Éphèse, Maladies des femmes, I, 12 [I, 39])7.

Par la réflexion et la pensée, par ses visions, par sa sensibilité et ses émotions, la mère imprime sa marque sur le bébé comme elle pourrait le faire en outre par son sang et/ou son lait. Les fluides ou humeurs féminines sont, en effet, considérés comme des vecteurs de transmission qui agissent sur les semences ou encore sur le nourrisson allaité - sur son apparence comme sur son caractère -, toutefois pas toujours de façon irréversible. C'est pourquoi, par exemple, le médecin athénien $d u \mathrm{IV}^{\mathrm{e}}$ siècle avant n. è., Mnésithée, cité par Oribase, médecin sous l'empereur Julien (IV siècle de n. è), recommande de choisir une nourrice parmi les proches ou parmi des femmes qui ressemblent pour leur forme (eidos) à la mère :

Nous préférons surtout les mères elles-mêmes (tas tetokuias autas), ou, si cela ne se peut pas, leurs proches (oikeias), leurs parentes (suggeneis), ou les femmes qui leur ressemblent pour la forme (tas homoias tautê ousas tôi eidei) (Oribase, Livres incertains, 15, 7-8 ; U. C. Bussemaker et C. Daremberg, CEuvres d'Oribase, III, [Paris, 1858], p. 130 ; voir Bertier 1972).

Les qualités positives (de beauté du corps, du caractère, du comportement) de la mère ou de celles avec lesquelles elle a des liens de familiarité ou de consanguinité passeraient ainsi à l'enfant (voir Dasen 2012, en particulier p. 57). À cet égard, on peut noter que la nourrice idéale doit être « de grande taille, la poitrine bien développée, les chairs d'une bonne nature et belle à voir (eumegethês, eupleuros, eusarkos, kalê tên opsin) ... » (Oribase, Livres incertains, 15, 2-3 ; Bussemaker et Daremberg, III, p. 130). En outre, en lien avec cette idée d'une influence du lait sur le développement physique du nourrisson, ce même médecin préconise qu'un « enfant allaité doit être de même sexe que celui du dernier enfant de la mère nourrice » (Oribase, Livres incertains, 15, 5-7 ; Bussemaker et Daremberg, III, p. 130 ; voir Dasen 2012, p. 57). Cela éviterait un détournement de l'identité sexuée : de féminiser un garçon ou bien de masculiniser une fille. Il y a donc bien relation/ communication et échange d'informations entre la mère et son enfant via le lait, une substance qui transmet des "propriétés » de la mère nourricière à son bébé. De même que la réflexion, les états d'âme et les impressions sensorielles de la mère, le lait sécrété est susceptible de modifier le caractère de l'enfant allaité. Ainsi, selon Soranos d'Éphèse, les bébés sont «de caractère paisible: parce que les nourrissons s'assimilent naturellement à celles qui les allaitent, devenant ainsi colériques du fait d'une nourrice irascible, ou doux et sages du fait d'une nourrice équilibrée » (II, 8, 87-89) ${ }^{8}$. Ce fluide féminin communique à l'enfant des caractéristiques maternelles ou de la nourrice, des signes génétiques et des états d'être ${ }^{9}$. La mère ou son substitut " nourrit » le bébé ${ }^{10}$ et le façonne suivant sa nature comme une bonne ou une mauvaise terre agit sur les plantes. 
Ce parallélisme avec le monde végétal « imprègne le discours hippocratique » relatif à la relation entre mère et fœtus/nourrisson (Dasen 2009, p. 44) :

Je dis que toutes les plantes vivent de l'humeur (humidité : ikmados) de la terre et que leur état dépend de celui de la terre par rapport à l'humeur. De même l'enfant vit de la mère dans la matrice et son état dépend donc de l'état de santé de la mère ( De la nature de l'enfant, XXVII, 1). les défauts physiques d'Agésilas, le second fils du roi Archidamos, qui, à Sparte, alors qu'il n'était pas destiné à devenir roi, régna $a u I^{\mathrm{e}} \mathrm{s}$ av. n. è. ?

Archidamos, fils de Zeuxidamos, après avoir régné glorieusement sur les Lacédémoniens, laissa un fils, Agis, qu'il avait eu de Lampidô, une femme

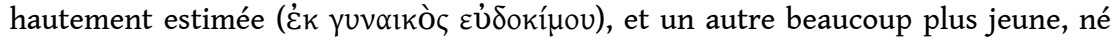
d'Eupolis, fille de Mélésippidas, Agésilas. [...] Quant à sa claudication, la beauté de

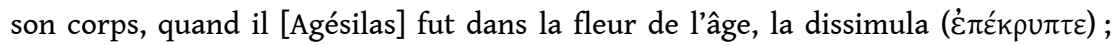
d'autre part, la facilité et la gaieté avec lesquelles il la supportait, étant le premier à en plaisanter et à se moquer de lui-même, contribuaient grandement à atténuer cette infirmité; elle rendait même son ardeur plus manifeste, car jamais il n'invoqua sa boiterie pour se dérober devant un effort ou une entreprise. Nous ne

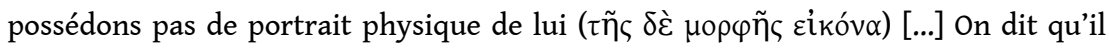

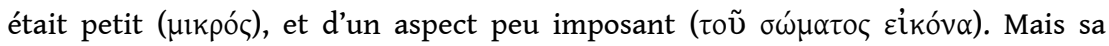
gaieté, sa bonne humeur en toutes circonstances, son enjouement, l'absence de toute morosité et de toute rudesse dans sa voix comme dans son regard, le rendirent dans sa vieillesse plus séduisant que les beaux jeunes gens dans la fleur de l'âge. Pourtant, d'après Théophraste, les éphores avaient condamné Archidamos à

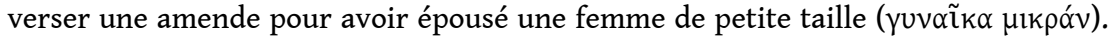
«Elle ne va pas nous donner des rois, disaient-ils, mais des roitelets ${ }^{11}$ ".

Dépourvu de beauté physique - un marqueur de l'aptitude à gouverner et à commander tel que le préconise la plupart des discours qui s'attachent à définir les qualités d'un bon dirigeant (voir Azoulay 2004) - Agésilas ne ressemble pas, en effet, par la conformation de son corps à un chef. À cet égard, sa petite taille plus que sa claudication en fait un être à l'aspect méprisable et, par conséquent, a priori inapte à la royauté. Toutefois, selon Plutarque, il surmonta cet handicap grâce aux qualités de son caractère et de son éducation qui firent oublier les tares physiques que lui avait transmises sa mère (du moins sa taille).

Dès lors, comment pourrait-on expliquer plus largement des défauts physiques comme ceux d'Agésilas, en suivant les théories de l'hérédité élaborées par les Anciens à l'époque classique? Si l'on suit Aristote: «De parents mutilés naissent des enfants mutilés, par exemple de boiteux naissent des boiteux, d'aveugles des aveugles, et en règle générale les enfants ressemblent aux parents pour les anomalies ; ils ont des marques semblables, par exemple des excroissances et des cicatrices [...] (Aristote, Histoire des animaux, VII, 6, 585a29-32 ; cf. aussi Génération des animaux, I, 17, 721b et 18, 724a3-7). En outre, d'après un auteur hippocratique, un enfant peut être estropié en raison d'une blessure consécutive à un choc qui se répercuterait sur le fœetus ou encore en raison d'une anomalie de l'utérus, 
par exemple de son étroitesse ou de sa mauvaise conformation. Dans ces derniers cas, la mère est directement en cause. Ainsi, dans De la génération, $\mathrm{X}, 2$ :

Si l'enfant est estropié dans la matrice, je dis que c'est à la suite d'une contusion, soit que la mère ait été frappée à l'endroit du foetus ou qu'elle soit tombée ou qu'elle ait subi tout autre violence [...]. Les enfants peuvent être estropiés de cette autre façon encore : si l'endroit de la matrice correspondant à telle articulation du fotus est étroit, il est fatal que, le corps se mouvant à l'étroit, le fotus soit estropié à cet endroit. C'est comme les arbres qui dans la terre n'ont pas d'espace, mais sont arrêtés par une pierre ou autre chose ...

Comme le note Véronique Dasen $(2009$, p. 44) « [t] oute anomalie du fotus est [...] liée à l'état utérin ", donc à la mère. Par son esprit comme par son corps, en particulier son utérus, la mère exerce une contrainte sur le corps du bébé. Cependant, dans le cas d'Agésilas, la claudication ne semble pas congénitale; seule sa petite taille l'est par le biais de sa mère.

A contrario, doit-on expliquer suivant ces conceptions la beauté de Mélinna louée dans un poème de l'Anthologie palatine?

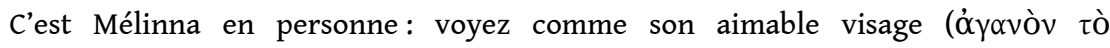
$\pi \rho$ ó $\omega \pi$ rov) semble nous regarder tendrement et comme tous les traits de la fille

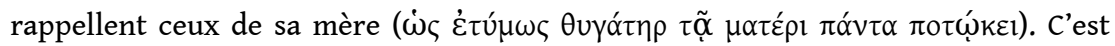

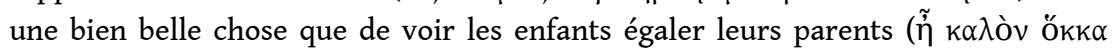

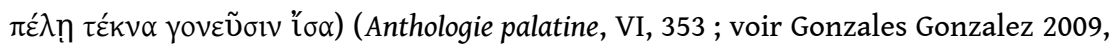
p. 120-121).

Il s'agit plutôt ici d'une illustration de l'aphorisme "telle mère telle fille ", qu'exploite également l'oniromancie. Ainsi, «rêver qu'on voit son image dans la lune, pour un homme sans enfant, cela prédit la naissance d'un fils, pour la femme la naissance d'une fille; car chacun des deux en effet verra une image pareille à lui-même, c'est-à-dire à un enfant » (Artémidore, Clef des songes, II, 36). Une relation de miroir existe entre parent et enfant de même sexe, l'un étant conçu comme l'exact reflet de l'autre (Frontisi-Ducroux et Vernant 1997, p. 120) - ce qui est conforme à la théorie d'Aristote (cf. supra). La beauté de l'un détermine par conséquent la beauté de l'autre.

\section{Transmission des comportements entre inné et acquis}

28 À la transmission de caractères physiques de la mère à son enfant est corrélée celle des comportements, suivant un schéma encore souvent «naturaliste ». La mère agit encore, en effet, sur l'attitude de ceux qu'elle porte puis élève, telle une bonne ou une mauvaise terre sur les fruits, comme l'attestent les exemples développés maintenant et concernant principalement la cité d'Athènes. Ceux-ci éclaireront comment, pour les Anciens, dans les représentations collectives, les manières d'être de la mère influent sur celles de sa fille et de son fils.

\section{De la mère à la fille}

Dans le corpus tragique athénien d'époque classique, le plus souvent, de même que le fils est le produit du père (Cf. Eschyle, Les Euménides, v. 658-661 ; voir Bonnard 2006, p. 307), la fille hérite du comportement de sa mère. Ainsi Hermione est victime des agissements répréhensibles d'Hélène. Comme le dit Pélée, souhaitant défendre les intérêts de son petit-fils, Néoptolème, et s'adressant en ces termes à Ménélas : 
Ah! Je le lui disais, moi, au futur époux, de ne pas s'allier à toi et de ne pas prendre chez lui le rejeton d'une coquine. Celles-là reproduisent au dehors les hontes maternelles. Avis à vous prétendants! Prenez fille de mère honnête.

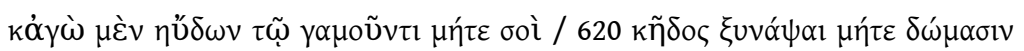

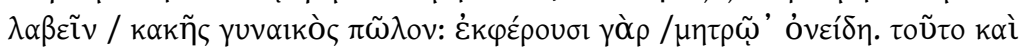

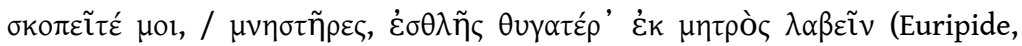
Andromaque, v. 619-624).

Hélène fut une mauvaise terre qui n'a pu produire (ekpherein) que de mauvais fruits. Qualités et défauts se transmettent de mère en fille. Les bonnes épouses engendrent de futures bonnes épouses contrairement à celles qui sont infidèles. On aurait ici un topos comme le suggère cette épigramme de l'Anthologie palatine (IX, 96) :

Antigénès de Géla dit un jour ces mots à sa fille, au moment de s'en aller dans l'Hadès, «Vierge aux belles joues, mon enfant, aie toujours pour compagne de travail la quenouille, bien qui suffit à une vie pauvre; et si tu viens à te marier,

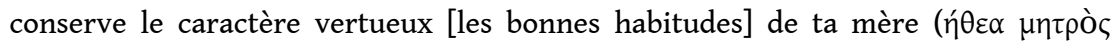

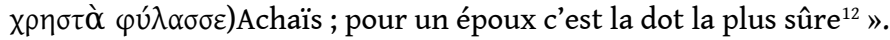

Bons et mauvais comportements (tropoi) relèvent des caractères de l'hérédité. Comme le souligne Andromaque, toujours à propos d'Hélène :

Celle qui te mit au monde a trop aimé les hommes; ne cherche donc point, femme, à aller plus loin qu'elle. Quand ils ont de mauvaises mères, les enfants doivent fuir

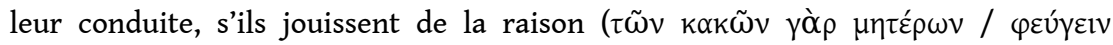

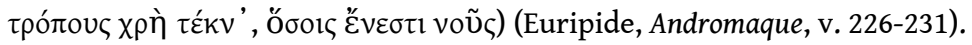

Dans cette perspective, Hermione, "fille de sa mère", est victime de son héritage maternel. Phèdre le serait également. Dans Hippolyte d'Euripide, aux vers 337-343, la fille de Minos, amoureuse de son beau-fils, remarque que sa propre mère Pasiphaé, éprise du taureau, et sa sœur Ariane, qu'épousa Dionysos, ont également subi les méfaits d'une passion dévorante : «la troisième à mon tour, je meurs, misérable » s'exclame-t-elle au vers 341 (voir Sourvinou-Inwood 1994). Des amours complexes et tourmentées sont communes à ces trois femmes: est-ce seulement le résultat d'une action divine (en l'occurrence de Cypris) ou cela relève-t-il davantage de l'hérédité ?

Si les filles, dans ce registre, ressemblent bien à leur mère, Électre, elle, tente de lutter contre cet "ordre naturel», se voulant et se proclamant du côté du père - les trois auteurs tragiques athéniens la mettant ainsi en scène. Aussi, dans les Choéphores, sur la tombe d'Agamemnon, demande-t-elle à son père défunt :

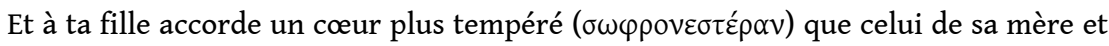

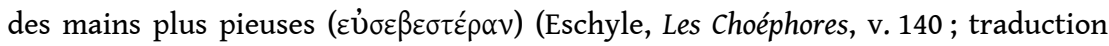
P. Mazon légèrement modifiée).

Pour ne pas reproduire les méfaits de Clytemnestre adultère et meurtrière, Électre se voudrait pleine de tempérance et de piété, refusant et niant ainsi la transmission maternelle. Dans le même ordre d'idée, dans la pièce éponyme de Sophocle, l'héroïne s'adresse en ces termes à Chrysothémis, sa sœur :

Mais alors que tu pourrais être appelée la fille du plus noble des hommes, va, faistoi appeler la fille de ta mère : tu auras l'air ainsi, aux yeux de presque tous, d'une mauvaise fille, qui a trahi son père mort, en même temps que tous les siens

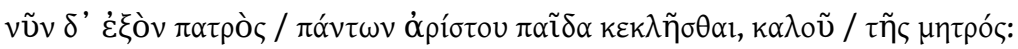

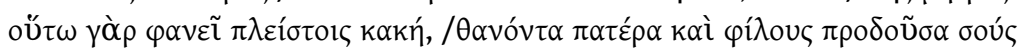
(Sophocle, Électre, v. 365-368). 
Il est de nouveau question de paideia à propos de la fille de Nééra dans le célèbre plaidoyer qui a été consacré à l'hétaïre. Phanô est décrite comme une fille qui a hérité des habitudes (ethos) et des dérèglements (akolasia) de sa mère. Pour ces raisons, alors qu'elle est mariée depuis un an et enceinte, son époux, Phrastor, décide de la renvoyer chez son père sans restituer la dot :

Elle ne sut pas se plier au caractère de son mari : ce qu'il lui fallait, c'était le train de sa mère et la vie dissolue qu'on menait chez elle, car elle avait été élevée, je suppose, avec la même licence. 51. Phrastor ne trouvait chez elle ni tenue ni docilité. De plus, il avait déjà acquis la certitude qu'elle n'était pas la fille de Stéphanos, mais de Nééra, et qu'il avait été trompé dès l'abord quand il l'avait reçue en mariage non pas comme étant de cette femme, mais comme fille légitime de Stéphanos qui l'aurait eue d'une épouse athénienne avant de vivre avec Nééra.

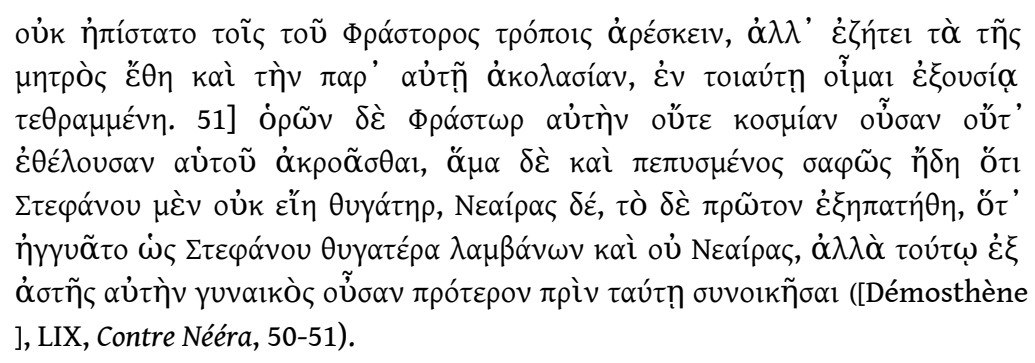

Phanô est bien la fille de sa mère. Son mauvais comportement (son absence d'ordre [ kosmos] ou le désordre de sa personnalité) et son indocilité en sont les indices. Ils traduisent ou révèlent ses origines, fruits d'une transmission certes par éducation mais quasi « héréditaire» du comportement que rien ne peut cacher. Ainsi, les mauvaises mères façonnent de mauvais enfants, comme une mauvaise terre produit de mauvais 
fruits. Discours tragiques et discours juridiques se rejoignent sur ce point, utilisant les mêmes arguments sans contredire, en outre, le discours médical et biologique ${ }^{17}$.

\section{Et de mère en fils ?} responsables de leur devenir d'adulte dans la cité. C'est précisément la thèse développée par Giulia Sissa dans une communication intitulée "The political intelligence of mothers" lors du colloque EUGESTA «Motherhood in Antiquity» (Manchester, avril 2014). Les mères, en effet, transmettent à leurs fils des valeurs politiques, sociales et religieuses; elles constituent, à cet égard, ou tentent d'être, des remparts contre la tyrannie ${ }^{18}$.

En revanche, dans un registre comique, le mauvais garçon est le fils de sa mère. Ainsi, dans les Nuées d'Aristophane, la mère de Phidippidès a d'abord choisi, en partie, le nom de son rejeton. De plus, elle est responsable, en raison d'une origine aristocratique et probablement de sa truphê, mais aussi en raison des soins et de l'éducation qu'elle lui a prodigués, des habitudes de celui-ci. «[P]ortant longue chevelure, [il] monte à cheval, conduit un attelage, rêve de chevaux", tandis que son père dépérit "voyant la lune ramener les jours de vingtaine", endetté à cause des loisirs dispendieux de son fils (Aristophane, Les Nuées, v.14-17). Comme le rappelle Stepsiade à ce dernier, lui, un

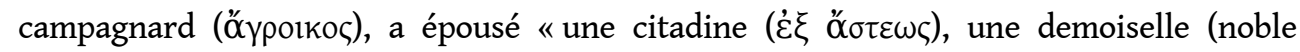

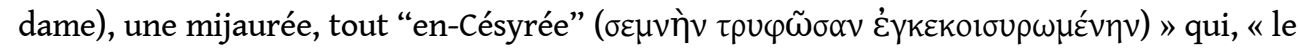
jour du mariage [...], sentait [...] les parfums, le safran, les baisers lascifs - la dépense, la gourmandise, Aphrodite Colias et Génétyllis » (Aristophane, Les Nuées, v. 39-55). Et, à la naissance de leur fils,

Elle voulut, pour lui, un nom aristocratique en « hippos »: Xanthippos ou Charippos ou Callippide ; moi, à cause du nom de son grand-père, je proposais Phidonidès [Parcimonieux]. Long fut le différend; enfin nous nous accordâmes pour l'appeler

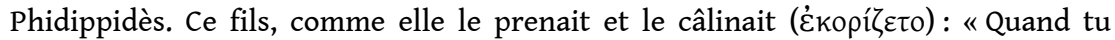
seras grand, lui disait-elle, et que tu conduiras ton char vers la cité, comme Mégaclès, vêtu d'une tunique de pourpre... » Moi je lui disais : "Quand, plutôt, tu ramèneras tes chèvres du mont Phellée, comme ton père, vêtu d'une peau de bique... » Mais il n'a pas voulu le moins du monde écouter mes paroles, et répandit une « chevalité » sur mes biens [...] (Aristophane, Les Nuées, v. 63-74).

Le mariage est hypogamique. La mère a fait de son enfant un cavalier et un citadin dépensier plutôt qu'un « cul-terreux » parcimonieux comme son père.

Enfin, dans les plaidoyers des orateurs attiques, la mère comme le père portent la responsabilité des mauvaises conduites de leurs fils. Néanmoins, dans les tribunaux démocratiques, la mère - prostituée, étrangère, indigente - de l'adversaire constitue pour les orateurs une «cible de choix, car l'invectiver permettait de mettre en doute la citoyenneté de son fils et de le faire ainsi sortir de la communauté civique [...]» (Villacèque 2014, § 3). 
est un phénomène culturel (Lett 1997, p. 17) qu'il s'agisse du corps comme des attitudes. Dans cette perspective, pour les Grecs d'époque classique, en particulier à Athènes, les mères, même si elles sont susceptibles d'imprimer de leurs propres caractères physiques leurs fils dans leur chair, semblent marquer plus durablement les filles en particulier en ce qui concerne l'accomplissement de leur destin de femme dans le mariage comme bonnes ou mauvaises épouses.

La maternité ne qualifie donc pas uniquement la capacité reproductrice des femmes ni leur aptitude supposée aux soins et à l'attention (tendresse) portés à leur progéniture (nouveau-nés et jeunes enfants) ${ }^{19}$. En ce sens, elle ne caractérise pas seulement une fonction relationnelle. Les mères en fonction de leur nature ont des qualités et des défauts physiques et psychiques qu'elles impriment sur leurs enfants - comme le ferait l'empreinte du poinçon (tupos) de l'artisan - , à l'état de fotus puis quand ils grandissent, les marquant dans leur corps comme dans leurs comportements ${ }^{20}$.

\section{BIBLIOGRAPHIE}

Azoulay V. (2004), Xénophon et les grâces du pouvoir. De la charis au charisme, Paris.

Berthier J. (1972), Mnésithée et Dieuchès, Leyde.

Billault A. (1981), « Le mythe de Persée et Les Éthiopiques d'Héliodore : légendes, représentation et fiction littéraire », REG 94, p. 63-75.

Boccara M. (1998), « Les tétons durs des nourrices font des enfants camus », dans Rey-Hulamm D. et Boccara M. dir., Odeurs du monde. Écritures de la nuit, Paris, p. 321-328.

Bodiou L. (2006), « De l'utilité du ventre des femmes. Lectures médicales du corps féminin », dans Prost F. et Wilgaux J. dir., Penser et représenter le corps dans l'Antiquité, Rennes, p. 153-166.

- (2011), « Les singulières conversions du lait maternel à l'époque classique. Approche médicale et biologique », Pallas 85, p. 141-151.

Bodiou L., Brulé P. et Pierini L. (2005), « En Grèce antique, la douloureuse obligation de la maternité », Clio. HFS 21, p. 17-42.

Bonnard J.-B. (2004), Le complexe de Zeus. Représentations de la paternité en Grèce ancienne, Paris.

- (2006), « Il paraît en effet que les fils ressemblent aux pères ", dans Prost F. et Wilgaux J. dir., Penser et représenter le corps dans l'Antiquité, Rennes, p. 307-318.

- (2013), « Corps masculin et corps féminin chez les médecins grecs », Clio. FGH 37, p. 21-39.

Dasen V. (2009), « Empreintes maternelles », dans La madre/ The mother, Micrologus XVII, Florence, p. 35-54.

- (2012), "Construire sa parenté par la nourriture à Rome », dans Dasen V. et Gérard-Zai M.-C. dir., Art de manger, art de vivre : nourriture et société de l'Antiquité à nos jours, Gollion (Suisse)-Paris, p. 40-59 et p. 240-249.

Cahiers « Mondes anciens », 6 | 2015 
Dean-Jones L. A. (2000), « Aristotle's Understanding of Plato's Receptacle and Its Significance for Aristotle's Theory of Familial Resemblance ", dans Wright M. R. éd., Reason and Necessity: Essays on Plato's Timaeus, Swansea, p. 101-112.

Djéribi M. (1988), « Le mauvais œil et le lait », L’Homme XXVIII, nº 105, p. 335-348.

Frontisi-Ducroux F. et Vernant J.-P. (1997), Dans l'œil du miroir, Paris.

Garland R. (1990), The Greek Way of Life from Conception to Old Age, Londres.

Gherchanoc F. (2015), « Naissance et rites de passage dans l'Athènes classique », dans Cid López R. M. dir., Parir y nacer en el Mediterráneo antiguo. Entre el mito y la historia, Vigo, [sous presse].

Gonzales Gonzalez M. (2009), « El lamento de las madres en los epitafios griegos: una Mirada a la Antología Palatina », dans Cid López R. M. dir., Madres y maternidades. Construcciones culturales en la civilización clásica, Oviedo, p. 115-127.

Gourevitch D. (1987), « Se mettre à trois pour faire un bel enfant ou l'imprégnation par le regard ", L'évolution psychiatrique LII, 2, p. 559-563.

HallE. (2006), The Theatrical Cast of Athens. Interactions between Ancient Greek Drama and Society, Oxford.

Henry D. (2006), « Understanding Aristotle's Reproductive Hylomorphism », Apeiron XXXIX/3, p. 257-287.

Laskaris J. (2008), « Nursing Mothers in Greek and Roman Medecine », AJA 112, 3, p. 459-464.

Loraux N. (1996), « Pourquoi les mères grecques imitent, à ce qu'on dit, la terre », Né de la terre. Mythe et politique à Athènes, Paris, p. 128-144.

Lett D. (1997), «L'“expression du visage paternel”. La ressemblance entre le père et le fils à la fin du Moyen Âge : un mode d'appropriation symbolique », CRM 4 [En ligne]. URL : http:// crm.revues.org/972 ; DOI : 10.4000/crm.972

Maire B. (2004), «L'imprégnation par le regard ou l'influence des "simulacres" sur l'embryon », dans Bianchi O. et Thevenaz O. éd., Mudry Ph. dir., Mirabilia. Conceptions et représentations de l'extraordinaire dans le monde antique, Berne, p. 279-294.

Roux O. (2009), « Parenté hippocratique et parenté aristotélicienne. Quelques réflexions sur les théories biologiques de la Grèce ancienne », Pallas 79, p. 307-322.

Saïd S. (1984), « La tragédie de la vengeance », dans Courtois G. dir., La vengeance. Études d'ethnologie, d'histoire et de philosophie, Paris, p. 47-90.

Sourvinou-Inwood C. (1994), « Le minotaure et les autres. Images et perceptions », Mètis IX, p. 227-235.

Svenbro J. (1988), Phrasikleia. Anthropologie de la lecture en Grèce ancienne, Paris.

Vernant J.-P. (1971 [1963]), « Hestia-Hermès. Sur l'expression religieuse de l'espace et du mouvement chez les Grecs », dans Mythe et pensée chez les Grecs I, Paris, p. 124-170.

Villacèque N. (2013), Spectateurs de paroles! Délibération démocratique et théâtre à Athènes à l'époque classique, Rennes.

- (2014), « Ta mère ! Insulte et généalogie à la tribune démocratique », dans Azoulay V. et Damet A. dir., Maudire et mal dire : paroles menaçantes en Grèce ancienne, Cahiers « Mondes anciens » 5 [En ligne]. URL : http://mondesanciens.revues.org/1242 ; DOI : 10.4000/mondesanciens.1242 
Wilson P. J. (1996), «Tragic Rhetoric: The Use of Tragedy in the Fourth-Century », dans Silk M. S. dir., Tragedy and the Tragic. Greek Theatre and Beyond, Oxford-New York, p. 310-331.

\section{NOTES}

1. Voir Lett 1997, § 17-18, pour la fin du Moyen Âge.

2. Sur les conceptions de l'hérédité chez les Présocratiques, Hippocrate et Aristote, voir entre autres Dean-Jones 2000 ; Bonnard 2004 et 2006 ; Roux 2009. Les théories présocratiques ne seront pas abordées dans cet article.

3. Sauf mention contraire, le texte suivi et les traductions adoptées sont ceux de la "Collection des Universités de France" publiée sous le patronage de l'Association Guillaume Budé aux Éditions Les Belles Lettres (Paris).

4. Sur l'« hylomorphisme », voir Henry 2006.

5. Voir le tableau établi à partir du texte d'Hippocrate par Bonnard 2004, p. 156 et par Roux 2009, p. 311.

6. Cette idée de «la femme imitatrice de la terre » est probablement empruntée à Platon; voir Loraux 1996, à propos du Ménéxène.

7. L'anecdote rapportée par le médecin d'Éphèse ne peut être contextualisée : on ignore qui fut ce tyran et à quelle date il gouverna (voir les notes 144 et 145, p. 83 de Maladie de femmes, I, Les Belles Lettres, Paris, 2003). Sur l'influence de la vision maternelle, voir Gourevitch 1987; Maire 2004 ; Dasen 2009, p. 40-41. Un récit comparable se trouve aussi, au II ${ }^{\mathrm{e}}$ s. de n. è., chez Galien (XIV, p. 253 sq. Kühn [De theriaca ad Pisonem II]) : «Un ancien récit m'a appris qu'un grand de ce monde, qui n'était pas beau mais voulait avoir un bel enfant, fit peindre sur une tablette de bois un joli jeune homme (eueides paidion) différent de lui, et que, tandis qu'il s'unissait à sa femme, il lui disait de regarder cette figure du tableau ", et au v siècle de $n$. è. chez un autre médecin, Caelius Aurelianus (Gynaecia, I, 50). Cette tradition relative à l'imprégnation par le regard, remonterait au $\mathrm{V}^{\mathrm{e}}$ siècle avant $\mathrm{n}$. è. et à Empédocle: «La conformation du fotus est soumise pendant la grossesse à l'imagination (phantasia) des femmes ; souvent elles se prennent d'amour pour des statues ou des tableaux et ont des enfants qui ressemblent à ces objets (morphousthai) » (H. Diels, Die Fragmente der Vorsokratiker, 21, A 81 in Aetius, V, 12, 21 (D 423) [Berlin, 1903]). Cf. aussi la fable que cite Denys d'Halicarnasse, Opuscules rhétoriques. Sur l'imitation [Traité IX], 1, 2, à propos de la ressemblance par imitation en littérature: «Un homme des champs avait, dit-on, un physique repoussant: il craignait de devenir le père d'enfants semblables à lui. Cette peur même lui enseigna l'art d'engendrer de beaux enfants (eupaidias). Il façonna des images de belle apparence (euprepeis), et fit prendre à sa femme l'habitude de les regarder. Après quoi il s'unit à elle et obtint que ses fils aient la beauté (to kallos) des images » ; cf. enfin Héliodore, Les Éthiopiques, IV, 8 , 3-5, au sujet de l'étonnante blancheur de peau de la belle Chariclée, fille du couple royal d'Éthiopie - résultat du modelage de la semence par une vision maternelle (voir Billault 1981, p. 63-68) ; sur l'influence des deux parents, cf. Pline l'Ancien, Histoire naturelle, VII, 52 : «La pensée même qui traverse brusquement l'esprit de l'un ou l'autre parent passe pour fixer ou altérer la ressemblance ». Dans d'autres cas, la nourriture ingérée par la mère agit, non la vision. Cf. Hippocrate, De la superfétation, 18 (Littré VIII 486, 7-9; Amsterdam, éd. A. M. Hakkert, 1982 [1853]) : «Si une femme enceinte a envie de manger de la terre ou des charbons et en mange, l'enfant qui est mis au monde porte sur la tête un signe provenant de ces choses ».

8. Cf. aussi Aulu-Gelle, Nuits attiques, XII, 19 (II s. de n. è) : "C'est ma foi, la raison pour laquelle nous nous étonnons bien souvent que certains enfants de femmes honnêtes se révèlent sans ressemblance ni de l'âme ni du corps de leurs parents "; car les qualités de la nourrice - bonnes ou mauvaises - sont transmises par son lait. D'ailleurs, d'après Aulu-Gelle : le « lait imprégné dès 
le début par l'agrégation de la semence paternelle, donne ensuite sa forme à la personnalité toute neuve d'après le corps et l'âme de la mère elle aussi » (XII, 1, 20).

9. De même, il transmet des maladies. Par exemple, selon Hippocrate, «si la nourrice n'est pas bien portante, mais qu'elle soit ou bileuse, ou humide, ou sanguine, ou pituiteuse, le lait devient mauvais pour l'enfant. [...] L'enfant, tétant un lait qui est non pas pur mais bilieux comme j'ai dit, devient maladif et faible, et cet effet dure tant que le lait tété est mauvais et morbifique " (Des Maladies, IV [Littré, VII, 55 ; Amsterdam, éd. A. M. Hakkert, 1979 [1851]). Le Stagirite se préoccupe lui aussi du lait maternel - de sa qualité nutritive dont les indices sont la couleur, l'odeur et la chaleur - duquel dépend la santé du nouveau-né: les «petits enfants sont sujets à des convulsions et en particulier ceux qui sont trop bien nourris, qui disposent d'un lait trop abondant et trop épais et dont les nourrices sont bien en chair " (Histoire des animaux, VII, 12). Outre Bodiou 2011, p. 146-147, voir Djéribi 1988 ; Boccara 1998 et Laskaris 2008.

10. Sur la « femme cuisinière et nourricière » de l'enfant, voir Bodiou 2006, p. 162-163.

11. Plutarque, Agésilas, I-II (traduction d'A.-M. Ozanam, Quarto Gallimard, Paris, 2001). Plutarque, Euvres morales, 1d (De l'éducation des enfants), se sert de la même histoire dans un développement relatif à la descendance et à l'importance de la bonne naissance (eugeneia) : « Les Lacédémoniens aussi méritent des louanges pour la noblesse dont ils firent montre en mettant à l'amende leur roi Archidamos, qui avait osé prendre en mariage une femme de petite taille, avec ce commentaire : "Ce ne sont pas des rois mais des roitelets qu'il envisage de nous donner" ".

12. La fille serait le «miroir de sa mère ». Ainsi, Kléis, la fille de Sappho, par son nom rappelle l'excellence, le « renom sonore » de sa mère. Voir Svenbro 1988, p. 170.

13. Cf. Eschyle, Choéphores, v. 135 ; Euripide, Électre, v. 107-110 ; 241 et 304-306 ; Sophocle, Électre, v. 616-621.

14. Cf. Euripide, Électre, v. 965-966; 1062-1064 et 1069-1075.

15. Sur l'opposition (l'une est « vierge » et « chaste », l'autre " plus sensuelle et dévergondée ») entre mère et fille comme sur le parallélisme de leur nature virile et dominatrice, voir Vernant 1971 [1963], p. 137-138; sur leur ressemblance avec répétition de scènes meurtrières contre des apparentés, voir Saïd 1984, p. 64 ; sur l'impossible transmission, voir Bodiou 2005, p. 32-36.

16. À cet égard, par exemple, comme le rappelle Ischomaque à propos de sa jeune épouse : «Pour

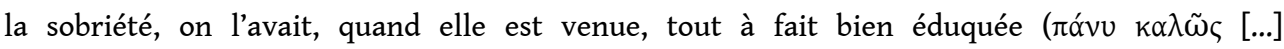
$\pi \varepsilon \pi \alpha 1 \delta \varepsilon \cup \mu \varepsilon ́ v \eta)$, or c'est là à mon sens, un point fort de l'éducation ( $\pi \alpha i ́ \delta \varepsilon u \mu \alpha)$ des hommes et des femmes » (Xénophon, Économique, VII, 6).

17. Sur l'articulation de ces discours et la façon dont ils se répondent ou complètent, voir notamment Wilson 1996 ; Hall 2006 ; Villacèque 2013.

18. Les exemples présentés concernaient notamment Jocaste, Aethra et Praxithéa.

19. Sur le rôle de la mère qui nourrit, soigne le nouveau-né, puis accompagne l'enfant jusqu'à un âge avancé, cf. Xénophon, Économique, VII, 24 ; voir Garland 1990, p. 147-158.

20. Je remercie Jean-Baptiste Bonnard pour sa relecture attentive de ce texte.

\section{RÉSUMÉS}

En confrontant des sources de différentes natures (textes médicaux et biologiques, tragédies et comédies, plaidoyers, poésie), cet article propose de réévaluer la part de la mère dans la construction de l'identité de l'enfant en Grèce ancienne, en particulier à l'époque classique. En 
d'autres termes, quelle est l'« empreinte » que laisse une mère sur ses enfants ? Leur transmetelle des caractères physiques et des comportements? Quel rôle joue-t-elle dans la détermination de leurs rôles sociaux et politiques?

My paper will examine a variety of sources (medical and biological texts, tragedy and comedy, forensic rhetoric, poetry), in order to consider and reassess the role of the mother in the construction of child-identity in the ancient Greek world, especially in classical time. In other words, what kind of 'imprint' does a mother put on her children? Does she transmit physical characteristics and behaviours to them? In what way does it affect their social and/or political roles during their life?

\section{INDEX}

Mots-clés : maternité, transmission, hérédité, identité de l'enfant, caractère physique, comportement, Grèce ancienne

Keywords : motherhood, transmission, heredity, child-identity, physical characteristic, behaviour, Ancient Greece

\section{AUTEUR}

\section{FLORENCE GHERCHANOC}

Université Paris Diderot-Paris 7, ANHIMA - UMR8210 